Recherches en didactique des langues et des cultures

Les cahiers de l'Acedle

16-1 | 2019

Enseigner la phonétique d'une langue étrangère

\title{
Enseignement et évaluation de la prononciation aujourd'hui : l'intelligibilité comme enjeu
}

Marion Didelot, Isabelle Racine, Françoise Zay et Alexei Prikhodkine

\section{OpenEdition}

Journals

Édition électronique

URL : http://journals.openedition.org/rdlc/4333

DOI : $10.4000 /$ rdlc.4333

ISSN : $1958-5772$

Éditeur

ACEDLE

Référence électronique

Marion Didelot, Isabelle Racine, Françoise Zay et Alexei Prikhodkine, «Enseignement et évaluation de la prononciation aujourd'hui : l'intelligibilité comme enjeu », Recherches en didactique des langues et des cultures [En ligne], 16-1 | 2019, mis en ligne le 30 janvier 2019, consulté le 20 avril 2019. URL : http:// journals.openedition.org/rdlc/4333; DOI : 10.4000/rdlc.4333

Ce document a été généré automatiquement le 20 avril 2019

\section{$\oplus \Theta \Theta$}

Recherches en didactique des langues et des cultures is licensed under a Creative Commons AttributionNonCommercial-NoDerivatives 4.0 International License 


\title{
Enseignement et évaluation de la prononciation aujourd'hui : l'intelligibilité comme enjeu
}

\author{
Marion Didelot, Isabelle Racine, Françoise Zay et Alexei Prikhodkine
}

Nous tenons à remercier les deux évaluateurs ainsi qu'Helene N. Andreassen, Sylvain Detey, Chantal Lyche et Roberto Paternostro de leurs commentaires avisés.

\section{Introduction}

1 La mondialisation et le contexte migratoire actuel contribuent à une augmentation sans précédent des échanges intergroupes et, de ce fait, des situations de communication exolingues. Une première conséquence de ces changements s'observe dans la modification des objectifs d'apprentissage d'une langue étrangère (ci-après L2 ${ }^{1}$ ), qui tendent désormais vers des dimensions clairement pratiques (Van Berten, 2016), telles que l'exercice d'une activité professionnelle ou l'intégration dans un nouveau pays d'accueil par exemple. Une deuxième conséquence concerne la façon dont la compétence langagière est considérée : de "simple » outil de communication, elle est peu à peu devenue un instrument de mesure de l'intégration (Hambye \& Romainville, 2014; HoganBrun, Mar Molinero \& Stevenson, 2009) et/ou de l'ascension socio-économique (Isaacs, 2016) d'un locuteur.

2 Même si les compétences liées à la maitrise de l'écrit restent très valorisées socialement et économiquement, la compétence orale est aujourd'hui davantage évaluée, et peut-être plus particulièrement dans le cas de locuteurs catégorisés comme non natifs. En effet, dans une majorité des États occidentaux, l'intégration des personnes migrantes - et de ce fait leur droit d'entrée et/ou de séjour - se mesure à travers l'évaluation de leur maitrise de la langue du pays d'accueil, évaluation qui prend fréquemment la forme d'entretiens oraux. Dans ce cas, le degré d'intégration d'un migrant est généralement apprécié sur la seule base d'une conversation orale avec un évaluateur, à l'issue de laquelle ce dernier décide si la personne s'exprime suffisamment bien pour pouvoir prétendre à un 
quelconque statut légal dans le pays d'accueil ${ }^{2}$. Si cet entretien évalue - ou du moins devrait évaluer - la performance orale globale du locuteur, soit la mise en œuvre de ses compétences phoniques, lexicales, (morpho)syntaxiques et pragmatiques (voir Fischer, 2007), on peut toutefois se poser la question du poids des aspects phoniques dans cette évaluation. En effet, comme le rappelle Jenkins (2000: 83), la prononciation constitue probablement «the greatest single barrier to successful communication »'. En d'autres termes, la compétence phonique d'un locuteur conditionne la réception du message qu'il veut transmettre et peut rendre difficile l'accès aux autres dimensions de la compétence orale évaluée. Le phénomène ne touche toutefois pas uniquement les personnes migrantes, la compétence orale est également hautement valorisée dans certains domaines professionnels (voir par exemple Grin, 1999). S'il est souvent nécessaire de savoir (bien) s'exprimer dans une voire plusieurs langue(s) étrangère(s) pour se voir offrir de nouvelles opportunités, la mobilité professionnelle accrue amène également les travailleurs à évoluer dans d'autres paysages linguistiques, où maîtriser oralement la langue locale permet une meilleure insertion socio-économique. Dès lors, au vu de l'importance particulière liée à la prononciation dans le contexte mondial actuel, il semble légitime de se questionner sur les enjeux liés tant à l'évaluation de la prononciation en L2 qu'à son enseignement en classe de langue.

Actuellement, le but affiché de l'enseignement de la prononciation - tel que promu par le CECRL (Cadre Européen Commun de Référence pour les Langues, Conseil de l'Europe, 2001) - est celui d'une parole dite « intelligible»: comme nous le verrons, cette notion demeurait jusqu'à récemment très vague et appelait à être mieux définie, notamment dans sa relation avec la notion de "force d'accent ${ }^{4}$, notion présente à la fois dans l'imaginaire collectif et les pratiques.

$4 \quad$ L'objectif de cette contribution est de proposer une réflexion épistémologique sur ces notions et les relations qu'elles entretiennent, au regard notamment de leur utilisation dans le descripteur de la compétence phonologique du CECRL, en en comparant l'ancienne (2001) et la toute nouvelle version (février 2018). Sur la base des motivations qui ont conduit à cette révision et des recherches menées pour l'anglais, nous formulerons ensuite quelques observations spécifiques au français.

\section{Intelligibilité, accent et CECRL}

Dans son article « Au-delà de l'identité», Brubaker (2001) critique le terme d'identité pour ses connotations essentialistes et son ambiguïté conceptuelle, et propose d'y substituer une série de termes analytiques plus univoques. Cette critique est, selon nous, également susceptible de s'appliquer aux termes d'intelligibilité et d'accent, dans la mesure où ils partagent, avec l'identité, au moins une caractéristique, celle d'être à la fois catégorie pratique dans l'usage quotidien et catégorie analytique dans le discours scientifique. Si, en sciences sociales, cette correspondance n'est pas rare, un manque de conceptualisation des termes d'intelligibilité et d'accent rend d'autant plus difficile, dans le travail scientifique, la rupture nécessaire avec le sens commun.

6 De par le caractère symbolique des langues naturelles, qui assure la fonction métalinguistique du langage, les significations linguistiques ne sont pas inhérentes à des mots ou à des manières de parler : elles sont l'objet de négociations, d'usages stratégiques et, en dernière analyse, de réflexivité (Coupland \& Jaworski, 2004: 15). Or, cette réflexivité - que l'on peut saisir à travers la notion de représentation sociale (Jodelet, 
1989) - a ceci de particulier que certaines significations peuvent s'imposer avec force au point de devenir une doxa, c'est-à-dire un "ensemble de croyances fondamentales qui n'ont même pas besoin de s'affirmer sous la forme d'un dogme explicite et conscient de lui-même»" (Bourdieu, 1997 : 30). Étant implicites et largement acceptées, les croyances doxiques n'ont pas besoin d'être justifiées : elles sont ressenties comme relevant du bon sens. Par conséquent, le danger pour le chercheur n'est pas tant une multitude de significations possibles attachées à un terme - cela pourrait, au contraire, l'inciter à en donner une définition explicite -, mais bien davantage l'existence de ces croyances doxiques, qui non seulement donnent l'illusion de ne pas devoir définir explicitement un concept mais empêchent également l'examen critique des significations paraissant évidentes.

Dans plusieurs travaux traitant de l'enseignement de la prononciation, l'intelligibilité et l'accent sont précisément des termes qui renvoient à cette illusion. Même si des travaux scientifiques visant à mieux définir ces notions ont été menés dans le monde anglo-saxon notamment (voir par exemple Derwing \& Munro, 2015 et Lippi-Green, 2012), force est de constater que ces recherches n'ont, jusqu'à très récemment, que peu influencé les pratiques. Le CECRL en donne un exemple significatif dans la première version de son descripteur de la maîtrise du système phonologique.

Tableau : Maîtrise du système phonologique selon le CECRL (Conseil de l'Europe, 2001 : 92).

\begin{tabular}{|l|l|}
\cline { 2 - 3 } & \multicolumn{1}{|c|}{ MAîTRISE DU SYSTÈME PHONOLOGIQUE } \\
\hline C2 & \multicolumn{1}{c|}{ Comme C1 } \\
\hline C1 & Peut varier l'intonation et placer l'accent phrastique correctement afin d'exprimer de fines nuances de sens. \\
\hline B2 & A acquis une prononciation et une intonation claires et naturelles. \\
\hline B1 & $\begin{array}{l}\text { La prononciation est clairement intelligible même si un accent étranger est quelquefois perceptible et si des erreurs de } \\
\text { prononciation proviennent occasionnellement. }\end{array}$ \\
\hline A2 & $\begin{array}{l}\text { La prononciation est en général suffisamment claire pour être comprise malgré un net accent étranger mais l'interlocuteur } \\
\text { devra parfois faire répéter. }\end{array}$ \\
\hline A1 & $\begin{array}{l}\text { La prononciation d'un répertoire très limité d'expressions et de mots mémorisés est compréhensible avec quelque effort } \\
\text { pour un locuteur natif habitué aux locuteurs du groupe linguistique de l'apprenant/utilisateur. }\end{array}$ \\
\hline
\end{tabular}

(C) CONSEIL DE L'EUROPE

8 À la lecture des exigences relatives à chaque niveau, on constate en effet que les termes compréhensible (A1), être compris (A2) et intelligible (B1) semblent être utilisés - sans être explicitement définis - de manière interchangeable et qu'ils disparaissent complètement dans les niveaux supérieurs. En outre, à nouveau sans définition explicite, les notions de clarté (claire en A2 et B2 et clairement en B1) et d'accent (net accent étranger en A2, accent étranger quelquefois perceptible en B1) s'immiscent dans le débat, la première semblant augmenter de manière inversement proportionnelle à la seconde. On peut relever également que la notion d'accent disparaît complètement à partir du niveau B2, où l'on voit tout à coup apparaître un élément suprasegmental isolé, l'intonation, qui est associée au qualificatif naturel. L'étude de Harding (2017), dans laquelle neuf évaluateurs expérimentés ont dû juger les productions orales de 44 apprenants d'anglais L2, illustre les difficultés rencontrées à manipuler, dans la pratique, ce descripteur. Les raisons principales invoquées par les évaluateurs sont d'une part le manque de définition des concepts utilisés et, d'autre part, le manque de cohérence entre les différents niveaux du descripteur. Enfin, on peut souligner que le recours à des adjectifs tels que clair et naturel constitue une stratégie argumentative répandue dans le discours idéologique, qui vise précisément à faire passer certaines croyances pour un point de vue universel et allant de 
soi, tout en masquant leur caractère socialement construit et arbitraire (voir par exemple Bourdieu, 1997 : 251 et Lipiansky, 1991 : 61). Les questions qui restent sans réponse, dans cette première version du CECRL, sont, en effet, celles de savoir qui décide de ce qui est « clair », « naturel » ou encore « intelligible » ou de ce qui ne l'est pas, sur quels critères et comment s'articulent les notions d'accent et d'intelligibilité.

La citation suivante de Lauret met en lumière l'association - restée implicite - entre ces deux notions: "[d]ans le cas d'un locuteur étranger, il s'agit a priori de chercher à être intelligible au plus grand nombre, c'est-à-dire de viser une production la moins marquée possible par un accent et la plus proche d'une prononciation 'modèle'», modèle qui "[...] représente le meilleur de la prononciation » (Lauret, 2007 : 18). Outre la clarification du lien de cause à effet entre force de l'accent et intelligibilité, cette dernière est ici présentée comme un argument permettant de cautionner l'imposition d'une variété légitime. Fait important, celle-ci est implicitement associée aux locuteurs natifs ou plutôt à une partie d'entre eux, à ceux dont le modèle langagier "[...] ne représente pas nécessairement la production du plus grand nombre, mais qui favorise l'intelligibilité» (Lauret, 2007 : 18). L'intelligibilité, ainsi présentée dans cette version du CECRL, joue le rôle de critère fondant la construction de la légitimité et contribue à essentialiser les pratiques langagières légitimes, car elle permet d'insister sur les «forces de la nature » (cognition) plutôt que sur les fondements socio-politiques de la légitimité. Recourir à ce critère permet de ne pas mentionner que le modèle langagier visé est en réalité celui des locuteurs natifs d'un certain niveau socioculturel, abstraction faite de la variété des usages réels.

10 En dernière analyse, il n'est pas déraisonnable d'avancer que l'usage de l'argument « intelligibilité » dans le discours sur l'enseignement de la prononciation du français L2, alors que ce critère reste flou, peut être un moyen de ne pas nommer explicitement le locuteur natif en tant que modèle (voir Rajagopalan, 2010, pour un constat similaire à propos de l'anglais). Depuis les années 1990, ce dernier modèle est critiqué dans la littérature scientifique pour sa charge idéologique et son inadéquation face à la diversité croissante de la population (voir Doerr, 2009, pour une synthèse), tandis que l'intelligibilité est promue au rang de critère fondant un modèle d'enseignement alternatif (Derwing \& Munro, 2015 : 6-7; Pickering, 2006 : 219-220). Cependant, comme le note Rajagopalan, mettre l'intelligibilité au centre du nouveau modèle ne suffit pas en soi pour changer de paradigme, car "[...] the figure of the native speaker creeps back in, only this time through the back door and that too most stealthily» $(2010: 468)^{5}$. En fait, dans la mesure où le locuteur natif peut encore difficilement figurer aujourd'hui comme modèle explicite, c'est l'intelligibilité qui devient un instrument de différenciation et, dans certains contextes d'exclusion sociale, car elle permet de porter des jugements prescriptifs de nature apparemment linguistique et objective, qui sont fondés, en réalité, sur un critère subjectif et discutable. Cette intelligibilité intuitive est un critère d'autant plus commode et plus acceptable qu'elle peut être perçue précisément comme socialement neutre et scientifiquement fondée. Et le manque de conceptualisation de l'intelligibilité contribue à cette construction idéologique, l'établissant en doxa qui n'a pas besoin d'être justifiée.

11 Faut-il pour autant renoncer aux termes «intelligibilité » et «force de l'accent » dans l'évaluation de la prononciation? Le fait que l'analyse scientifique partage le même terme que l'usage quotidien n'est pas en soi une raison suffisante pour l'abandonner. Comme le souligne Brubaker, "[c]e qui est problématique, ce n'est pas le fait qu'un terme particulier soit employé, mais la manière dont il est employé » (2001: 70). Le volume complémentaire du 
CECRL avec ses nouveaux descripteurs répond à une grande partie des interrogations que nous posions à l'issue de l'examen de la première version. L'échelle conçue pour la phonologie s'étant en effet révélée «la moins réussie des échelles calibrées de la recherche originale » (Conseil de l'Europe, 2018: 140), une réflexion conséquente, impliquant notamment des chercheurs reconnus dans le domaine, a été menée, réflexion qui a abouti à une version complètement remaniée et sensiblement plus développée du descripteur de la compétence phonologique (voir le rapport de Piccardo, 2016, qui expose en détail la genèse de ce nouveau descripteur). Quatre domaines ont été identifiés - articulation, prosodie, accent et intelligibilité - aboutissant, comme on le voit (cf. Tableau 2) à une concrétisation, en termes d'échelles, en trois catégories : maitrise générale du système phonologique - qui remplace l'échelle de 2001-, articulation des sons et traits prosodiques. Si l'accent est mentionné, dans la première de ces catégories, à tous les niveaux du CECRL, on peut néanmoins regretter le manque de réflexion poussée autour de l'utilisation de ce terme dans la traduction en français. En effet, le mot « accent » est utilisé indistinctement pour indiquer, aux niveaux A1 et A2, le fait de rendre saillante une syllabe donnée d'un mot - traduction, en version originale, du terme «stress » - et, de B1 à $\mathrm{C} 2$, pour désigner la parole accentuée - « accent » en anglais.

Tableau : Maîtrise du système phonologique selon le CECRL (Conseil de l'Europe, 2018 : 142)
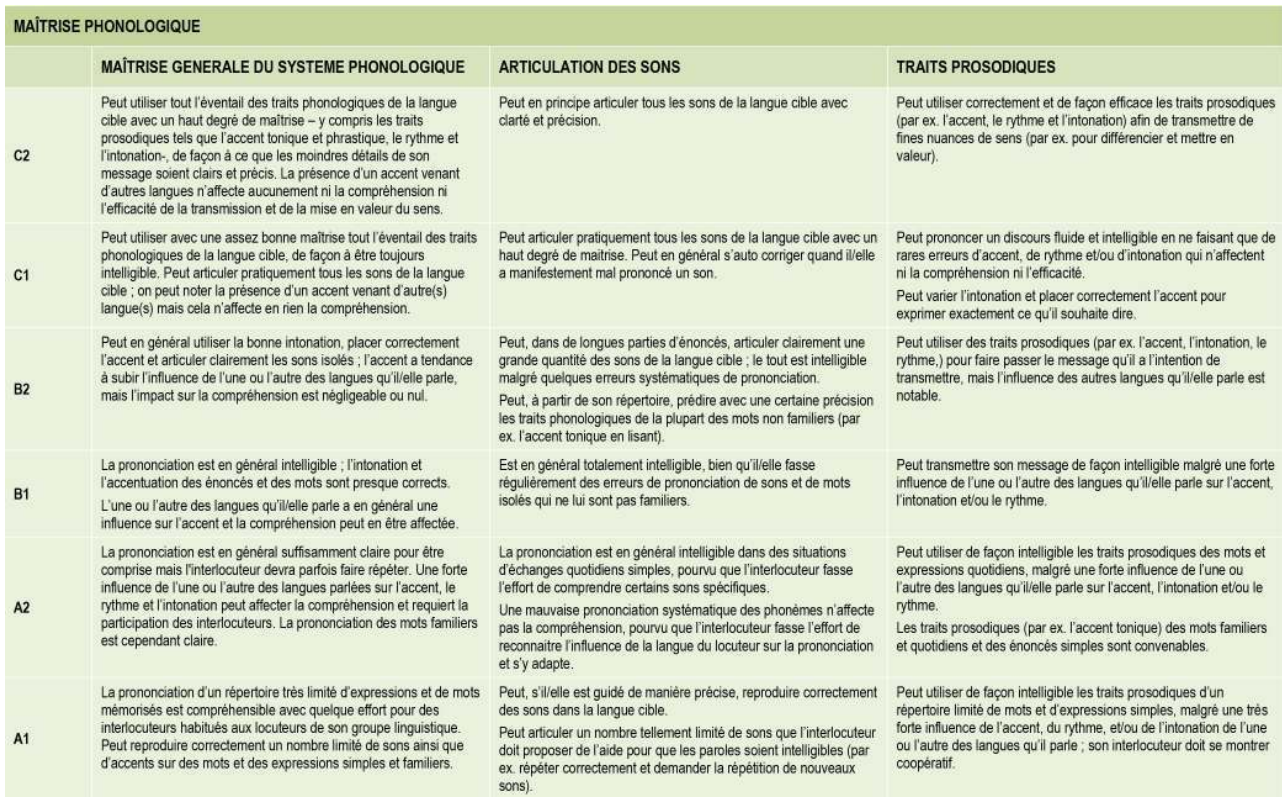

(C) CONSEIL DE L'EUROPE

12 Si l'on peut relever une volonté manifeste, dans le texte d'accompagnement, d'esquisser des définitions des notions clés utilisées, le changement le plus remarquable se situe indubitablement au niveau du modèle à atteindre, qui n'est plus le locuteur natif : ce changement de perspective complet répond de manière heureuse à la critique la plus virulente que nous avons formulée ci-dessus à l'encontre de la version de 2001. Les auteurs soulignent en effet que "l'insistance mise sur l'accent et son exactitude au détriment de l'intelligibilité a nui au développement de l'enseignement de la prononciation » puisque «les modèles idéalisés qui ignorent les accents ne prennent pas en compte les contextes, les aspects sociolinguistiques ni les besoins des apprenants » (2018: 140). Le rôle de l'accent et sa relation avec l'intelligibilité sont, de ce fait, plus explicites puisque, outre une coprésence, à tous 
les niveaux sauf en A1, de la notion d'intelligibilité et de celle de force de l'accent, sans présence d'un rapport inversement proportionnel, on observe également, en C2, qu'un accent qui n'affecte pas le message est maintenant accepté dans le descripteur. Les compétences plurilingues du locuteur sont par conséquent valorisées par ce changement de paradigme puisque l'influence des autres langues qu'il parle est maintenant admise, y compris au niveau C2. Il est intéressant de constater que cette dissociation entre force de l'accent et intelligibilité n'est pas nouvelle puisqu'on la trouvait déjà chez Abercrombie (1949), qui soulignait le fait que les apprenants ne devaient pas viser une prononciation identique à celle d'un natif ${ }^{6}$ mais une parole intelligible.

La définition proposée pour cette intelligibilité, qui est vue comme "l'effort que doit déployer l'interlocuteur pour décoder le message du locuteur » (2018:141) constitue également un changement majeur dans cette nouvelle version du CECRL, puisqu'elle place de facto l'interlocuteur-auditeur au centre du processus. Ceci étant, le clivage observé dans la version de 2001, qui lui attribuait un rôle central dans les trois premiers niveaux pour ne se focaliser que sur le seul locuteur à partir de B2, n'a pas complètement disparu dans la nouvelle version. La notion d'intelligibilité, mentionnée à tous les niveaux, y intègre de facto l'activité décodante de l'auditeur, mais un examen plus détaillé révèle que la participation de l'interlocuteur n'apparait explicitement qu'en A1 et A2, à la fois pour la maîtrise générale (A2: une forte influence de l'une ou l'autre des langues parlées [...] requiert la participation des interlocuteurs), pour l'articulation des sons (A2: [...] pourvu que l'interlocuteur fasse l'effort de reconnaitre l'influence de la langue du locuteur sur la prononciation et s'y adapte et [...] pourvu que l'interlocuteur fasse l'effort de comprendre certains sons spécifiques) et pour les traits prosodiques (A1: son interlocuteur doit se montrer compréhensif ). Il nous paraît d'autant plus important, pour définir la notion d'intelligibilité, de tenter de mieux cerner en quoi consiste cet « effort » requis de la part de l'auditeur, et comment en mesurer les degrés.

\section{Intelligibilité, entre dimensions linguistique et sociale}

Pour Horner (2014: 114), si le caractère inintelligible - c'est-à-dire particulièrement difficile à décoder - de certains énoncés constitue un frein à la communication, celui-ci peut être vu certes en termes de prononciation erronée du locuteur, mais également de compétence d'écoute déficiente de l'auditeur. On peut comprendre cette notion de " compétence d'écoute » à la fois comme la capacité à assouplir ou à élargir les catégories dans lesquelles intégrer la réalité acoustique perçue pour l'interpréter dans son système phonologique et comme une attitude plus ou moins coopérative envers l'interlocuteur. Un consensus se dégage par ailleurs des travaux de plusieurs chercheurs (entre autres Derwing \& Munro 2009, 2015 ; Didelot \& Racine, sous presse ; Field, 2005 ; Levelle \& Levis, 2014 ; Pickering, 2006), celui de considérer l'intelligibilité comme co-construite entre locuteur et auditeur, c'est-à-dire comme un phénomène perceptif dont la mesure, à l'instar de l'accent, implique la prise en compte du jugement de l'auditeur et qui peut être défini comme un degré de compréhension par un auditeur de ce qu'un locuteur a dit (Derwing \& Munro, 2015: 178). La question qui reste centrale est de déterminer précisément ce qui rend, aux «oreilles » de l'auditeur, une parole non native plus ou moins intelligible. Selon les premières études (voir Pickering, 2006, pour une revue), il semblerait que deux types de facteurs peuvent avoir une incidence sur la perception de l'intelligibilité : linguistiques, d'une part, et sociaux d'autre part. 
Les facteurs linguistiques concernent la performance phonético-phonologique des apprenants et peuvent être abordés à travers la notion de rendement fonctionnel. Comme l'expliquent Derwing et Munro (2015), il existe, dans chaque langue, des paires de phonèmes plus «importantes » que d'autres et ayant un impact plus marqué pour la compréhension, ce qui leur confère un « rendement » plus élevé. Le calcul de ce dernier s'effectue en prenant en compte le nombre de mots qui se distinguent uniquement grâce à cette opposition entre deux phonèmes, la fréquence des occurrences qui composent ces paires minimales et la catégorie syntaxique des mots dans les paires. Ainsi, pour reprendre les exemples de Derwing et Munro (2015) pour l'anglais, l'opposition entre /p/ et $/ \mathrm{b} / \mathrm{a}$ un rendement fonctionnel plus élevé, au vu du nombre de mots se distinguant grâce à ces deux sons (pet-bet, pea-bee, pate-bait, etc.), que l'opposition entre / $\theta /$ et /ð/, qui ne concerne que peu de mots (thigh-thy). Le rendement fonctionnel d'une paire minimale se verra également renforcé lorsque les deux mots de la paire sont très fréquents, entrainant un plus grand risque de confusion, alors qu'il sera moindre si l'une des deux occurrences est rare et encore plus faible si les deux occurrences sont peu communes. En suivant ce raisonnement, comme le soulignent les auteurs, il semble plus probable qu'un auditeur confonde un adjectif avec un autre adjectif qu'avec un substantif ou un verbe, ce qui confère aux paires minimales de la même catégorie syntaxique un rendement fonctionnel plus élevé. Si le rendement fonctionnel peut être prédit et calculé grâce à une analyse minutieuse du système de la langue, Derwing et Munro (2015) soulignent toutefois la nécessité de tester ces prédictions grâce à des études de perception auprès d'auditeurs naïfs afin de déterminer si une prononciation erronée des paires minimales dites à haut rendement fonctionnel perturbe réellement plus la compréhension que celle d'une paire minimale dont le rendement fonctionnel est estimé très faible (voir l'étude de Munro \& Derwing, 2006, pour un aperçu). Il parait également important de souligner que, si cette notion de rendement fonctionnel en termes de paires minimales ne s'applique qu'au niveau segmental, l'instauration d'une hiérarchisation des différents éléments de prononciation permet quant à elle de prendre également en compte la dimension suprasegmentale, qui fait partie intégrante de la prononciation, comme l'illustre la troisième partie du nouveau descripteur du CECRL.

16 Si cette perspective permet d'apporter un degré de validité supérieur aux mesures de l'intelligibilité et constitue, à ce titre, un critère au fort potentiel pour l'enseignement/ apprentissage, il faut néanmoins souligner quelques difficultés majeures. En effet, comme nous l'avons mentionné, elle est non seulement tributaire de descriptions linguistiques fines et doit être complétée par un volet perceptif, mais elle requiert également un investissement conséquent en termes de formation des enseignants amenés à se servir de ces ressources. Une autre difficulté réside dans le fait qu'elle demande de considérer une langue cible à la fois et, si un travail conséquent a déjà été mené pour l'anglais, c'est nettement moins le cas pour les autres langues ${ }^{7}$, ce qui explique sans doute le peu de généralisations possibles dans l'état actuel de la recherche. En effet, force est de constater, à la lumière des éléments-clés en termes d'intelligibilité pour l'anglais proposés par Horner (2014: 113) sur la base d'une synthèse de nombreux travaux empiriques $^{8}$, que ces éléments ne sont en aucun cas directement applicables au français, par exemple.

17 Le deuxième type de facteurs - auquel l'étiquette « social » peut être attribuée - a trait à l'auditeur et, plus généralement, au contexte de l'interaction. Parmi ces facteurs, on range principalement la familiarité de l'auditeur avec des variétés linguistiques ainsi que 
les représentations attachées à des groupes sociaux. L'incidence de la familiarité sur le degré de compréhension d'un énoncé a été montrée dans les années 1980 déjà par l'étude pionnière de Gass et Varonis (1984) portant sur la parole non native. Selon les auteurs, quatre types de familiarité contribuent à la compréhension : la familiarité avec le sujet de discussion; celle avec la parole non native en général ; celle avec un accent non natif en particulier ; et celle avec un locuteur non natif en particulier. Leurs résultats ont montré que le premier type de familiarité (avec le sujet de discussion) est celui qui contribue le plus à augmenter le degré de compréhension d'un locuteur non natif, mais ont également laissé entrevoir le bénéfice des trois autres catégories. Une étude plus récente de Derwing, Rossiter et Munro sur l'évaluation de la parole non native par de futurs travailleurs sociaux (2002) arrive à des conclusions similaires. En effet, les auteurs ont montré qu'avec une sensibilisation d'une dizaine de semaines à l'interculturalité et à la parole accentuée en général, les auditeurs évaluaient de manière significativement plus tolérante qu'auparavant des extraits de parole non native, alors que ce n'était pas le cas du groupe contrôle n'ayant reçu aucune formation spécifique.

Si la dimension liée à la familiarité avec un accent a fait une timide apparition dans le nouveau descripteur, avec la mention, au niveau A1 de la maîtrise générale du système phonologique, d'interlocuteurs habitués aux locuteurs de son groupe linguistique, celle des représentations sociales est quant à elle totalement absente du descripteur. Or, l'influence de ces dernières illustre très bien le caractère socialement construit de l'intelligibilité et montre qu'elle n'est que partiellement objective sur le plan linguistique. L'incidence des représentations attachées à des groupes sociaux peut être saisie à travers la notion de reverse linguistic stereotyping (Kang \& Rubin, 2009 ; voir Prikhodkine \& Correia Saavedra, 2016, pour une synthèse), qui désigne des situations où les stimuli linguistiques deviennent des objets de stéréotypes activés par des indicateurs non-verbaux d'appartenance catégorielle. Par exemple, lors d'une expérience portant sur la perception des compétences langagières des assistants dans une université américaine, Rubin (1992) a montré que les étudiants perçoivent un accent étranger et comprennent moins bien un assistant s'exprimant pourtant dans la variété standard de l'anglais américain, lorsqu'on leur fait penser, par le biais d'une photo, que cet assistant est d'origine asiatique. Par la suite, Rubin et ses collaborateurs ont, dans différentes études (voir Kang \& Rubin, 2009, pour une synthèse), démontré l'existence d'une certaine corrélation entre le degré d'intelligibilité perçue et la convenance attribuée à certains postes de travail et ce, à la fois pour des locuteurs natifs et non natifs, conclusions auxquelles parviennent également Didelot \& Racine (sous presse) pour le français. Ainsi, leurs résultats d'une part confirment, pour le français, l'absence d'une corrélation étroite entre force d'accent et intelligibilité. D'autre part, en montrant qu'à force d'accent jugée égale, certaines locutrices sont estimées plus intelligibles que d'autres et convenant mieux pour l'ensemble des postes soumis à évaluation, ils suggèrent que l'appréciation de l'intelligibilité comporte une part de subjectivité liée à l'évaluation socio-économique du locuteur. Il serait néanmoins erroné de considérer l'intelligibilité comme une construction purement sociale et dépourvue de toute dimension linguistique, les deux dimensions étant inhérentes à l'appréciation de l'intelligibilité. 


\section{Quid pour le français ?}

$19 \mathrm{Si}$, comme nous l'avons vu dans la section précédente, le fait que la dimension linguistique liée à l'intelligibilité soit spécifique à chaque langue semble une évidence puisque, à l'image d'une recette de cuisine, les différents ingrédients phoniques qui la composent - ainsi que leur dosage - sont uniques, il en va de même pour sa dimension sociale. Nous proposons maintenant de transposer cette réflexion au français.

Concernant la première dimension, très peu d'études ont tenté, à notre connaissance, de déterminer, pour le français, les corrélats linguistiques de l'intelligibilité (éléments segmentaux et suprasegmentaux sur lesquels se basent les auditeurs pour déterminer le caractère intelligible ou non d'un énoncé). L'étude pilote de Trottier (2007) permet néanmoins de confirmer la forte dépendance entre la dimension linguistique de l'intelligibilité et les caractéristiques phoniques spécifiques à une langue donnée, puisque les critères globaux utilisés par les évaluateurs sont différents des critères mis en avant pour l'anglais par Horner (2014: 113; voir supra). Pour préciser et hiérarchiser les éléments de prononciation qui jouent un rôle déterminant dans l'intelligibilité du français, il semble intéressant de développer la notion de rendement fonctionnel (Derwing \& Munro, 2015), en l'adaptant aux spécificités segmentales du français. Ainsi, par exemple, l'opposition / $\tilde{\varepsilon} /-/ \tilde{a} /$ a un rendement fonctionnel élevé en français, au vu du nombre de mots qu'elle permet de distinguer (vin-vent, teint-tant/temps, enfin-enfant, etc.), de même

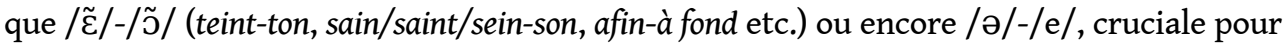
la distinction singulier/pluriel (le livre vs les livres) ainsi que dans la morphologie verbale (

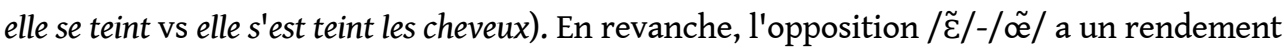
fonctionnel moins élevé, ce qui a d'ailleurs conduit à la disparition de / ̃̃/ dans un grand nombre de variétés (Lyche, 2010). Mentionnons également que, bien que la réalisation d'un /R/ apical ([r]) soit très souvent stigmatisée, elle n'est en aucun cas fonctionnelle, le signifié n'étant pas modifiée par cette réalisation. Elle renvoie en revanche à la problématique de la norme, sur laquelle nous reviendrons ci-dessous. En outre, même si la dimension suprasegmentale peut, de prime abord, paraitre moins importante qu'en anglais puisque, en français, la notion d'accent de mot - au sens d'une syllabe saillante à l'intérieur d'un mot - n'est pas distinctive, le placement des syllabes accentuées en fin de groupes rythmiques combiné à une courbe intonative qui s'affranchit aussi des limites des «mots » permet, en français, de structurer l'énoncé et pourrait, à ce titre, jouer un rôle déterminant au niveau de l'intelligibilité. De nombreux travaux doivent donc être menés sur le français afin, dans une première étape, de déterminer les éléments dont la réalisation erronée est susceptible de provoquer un coût, de les hiérarchiser et ensuite, dans une seconde étape, d'en tester la perception par des auditeurs naïfs.

La dimension sociale de l'intelligibilité est elle aussi susceptible de varier sensiblement en fonction de la langue, notamment en raison du rapport à la norme. Si l'anglais peut être vu comme polycentrique, le français est quant à lui considéré comme très monocentrique (Pöll, 2005), au vu de la stigmatisation de tout écart à la norme, y compris ceux commis par des locuteurs natifs (Houdebine-Gravaud, 2017). Cette attitude est directement observable dans les résultats de plusieurs études menées au Canada. Ainsi, Kennedy, Blanchet \& Guénette (2017), qui ont examiné l'évaluation de la prononciation d'apprenants de FLE par des enseignants de français au Québec, soulignent le caractère implicite de cette évaluation, en raison notamment de l'absence de définition claire de la 
- ou des - norme(s) de prononciation dans l'enseignement du FLE. Cette lacune se traduit également, dans l'étude de Lavoie \& Bouchard (2017) - où de futurs enseignants francophones natifs québécois ont dû autoévaluer leur propre compétence orale -, par une forte stigmatisation de certains traits spécifiques aux variétés québécoises pourtant décrits dans la littérature (voir Côté, 2016). Comme le souligne Lyche (2010: 160), la question de la norme pour le français oral renvoie finalement elle aussi à une doxa puisque ce français de référence n'a pas de « véritable ancrage dans une variété de langue bien définie ». L'indispensable travail visant à décrire les différentes variétés de français dans l'optique de mieux définir des normes de prononciation propres aux différents espaces francophones a été entamé depuis plusieurs années déjà dans le cadre du programme "Phonologie du Français Contemporain» (PFC, voir notamment Detey, 2010, 2017 ; Detey, et al., 2010, 2016a ; Guess, Lyche \& Meisenburg, 2012) ${ }^{9}$.

\section{Conclusion}

Nous l'avons vu, la notion d'intelligibilité ne se laisse pas appréhender à travers une seule dimension et des travaux spécifiques pour le français doivent être menés tant dans l'une des dimensions que dans l'autre. Ils doivent également tenter de comprendre comment interagissent les facteurs linguistiques et sociaux. Une manière de concevoir une telle interaction serait d'intégrer les recherches sur le style sociolinguistique. Cette notion postule qu'une variante linguistique n'a pas une signification sociale fixe mais se définit à l'intérieur d'ensembles de variables (les styles) faisant partie d'un système de distinction (Irvine, 2001 ; Eckert, 2008). Comme Coupland (2007 : 23-34) le suggère, la signification sociale de ces variantes peut être vue comme un potentiel pouvant être activé, validé ou remis en question. De cette façon, un même élément linguistique peut apparaître dans différents styles, en véhiculant des significations différentes. En l'appliquant à notre propos, un élément segmental ou prosodique n'aurait ainsi pas un «coût» fixe et déterminé à l'avance pour l'intelligibilité. Le fait qu'il puisse avoir une incidence sur l'intelligibilité serait ainsi dû à des indices contextuels, qui peuvent être autant verbaux que non-verbaux. S'agissant des indices verbaux, l'effet sur la perception de l'intelligibilité pourrait être différent selon qu'un élément fait partie de tel ou tel ensemble de pratiques perçu comme un style distinctif («accent espagnol», «accent albanais ", " accent marseillais », etc.). Par exemple, la présence d'un appendice nasal

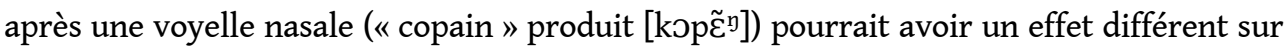
l'intelligibilité selon que cette consonne est perçue, grâce à d'autres éléments phonétiques présents dans le discours, comme constitutive d'un accent méridional, d'un accent hispanophone ou japonophone. En ce qui concerne les indices non-verbaux, le «coût» d'un élément sur l'intelligibilité pourrait aussi varier en fonction de l'information sociale sur le locuteur et le style, fournie par des indicateurs non-verbaux d'appartenance sociale (cf. reverse linguistic stereotyping).

23 Ainsi, comme nous l'avons vu, évaluer la prononciation s'avère être une opération dans laquelle interviennent des paramètres linguistiques et sociaux qui interagissent de façon dynamique. Cette complexité contraste avec la rapidité et l'apparente simplicité des jugements intuitifs émis sur la façon de «bien prononcer». Si l'on souhaite atténuer la part subjective de cette évaluation, il s'agira de repenser la formation dans ce domaine, en transposant les acquis de la recherche pour interroger la doxa et en y intégrant une 
sensibilisation à l'incidence des représentations sociales sur la perception de la performance des locuteurs.

\section{BIBLIOGRAPHIE}

Abercrombie, D. (1949). Teaching Pronunciation". English Language Teaching, vol. 3, pp. 113-122.

Bourdieu, P. (1997). Méditations pascaliennes. Paris : Éditions du Seuil.

Brubaker, R. (2001). «Au-delà de l'« identité ». Actes de la recherche en sciences sociales, vol. 139, pp. 66-85.

Conseil de l'Europe (2018). Cadre européen commun de référence pour les langues : apprendre, enseigner, évaluer. Volume complémentaire avec de nouveaux descripteurs. Disponible en ligne. https:// rm.coe.int/cecr-volume-complementaire-avec-de-nouveaux-descripteurs/16807875d5 (Consulté le 28 juin 2018).

Conseil de l'Europe (2001). Cadre européen commun de référence pour les langues : apprendre, enseigner, évaluer. Disponible en ligne. https://rm.coe.int/16802fc3a8 (Consulté le 26 juin 2018).

Côté, M.-H. (2016). « Le français au Canada ». In Detey, S., Racine, I., Kawaguchi, Y. \& Eychene, J. (dir.). La prononciation du français dans le monde : du natif à l'apprenant. Paris : CLE International, pp. 49-53.

Coupland N. (2007). Style: Language Variation and Identity. New-York: Cambridge University Press.

Coupland, N. \& Jaworski A. (2004). "Sociolinguistic Perspectives on Metalanguage: Reflexivity, Evaluation and Ideology". In Jaworski, A., Coupland, N. \& Galasinski, D. (dir.), Metalanguage. Social and Ideological Perspectives. Berlin: Mouton de Gruyter, pp. 15-51.

Derwing, T. M. \& Munro, M. J. (2015). Pronunciation Fundamentals: Evidence-Based Perspectives for L2 Teaching and Research. Amsterdam: John Benjamins.

Derwing, T. M. \& Munro, M. J. (2009). "Putting Accent in its Place: Rethinking Obstacles to Communication". Language Teaching, vol. 42, $\mathrm{n}^{\circ} 4$, pp. 476-490.

Derwing, T. M., Rossiter, M. J. \& Munro, M. J. (2002), "Teaching Native Speakers to Listen to Foreign-Accented Speech". Journal of Multilingual and Multicultural Development, vol. 23, 245-259.

Detey, S. (2017). «La variation dans l'enseignement du français parlé en FLE : des recherches linguistiques sur la francophonie aux questionnements didactiques sur l'authenticité ». In Jeng, A.-C., Montoneri, B. \& Maitre, M.-J. (dir.). Echanges culturels aujourd'hui : langue et littérature. New Taipei City : Tamkang University Press, pp. 93-114.

Detey, S. (2010). « Normes pédagogiques et corpus oraux en FLE : le curseur apprenabilité/ acceptabilité et la variation phonético-phonologique dans l'espace francophone ». In Bertrand, 0 . \& Schaffner, I. (dir.). Quel français enseigner ? La question de la norme dans l'enseignement/ apprentissage. Paris : Editions de l'Ecole Polytechnique, pp. 155-168.

Detey, S., Durand, J., Laks, B. \& Lyche, C. (2016a). Varieties of Spoken French. Cambridge: Oxford University Press. 
Detey, S., Lyche, C., Racine, I., Schwab, S. \& Le Gac, D. (2016b). "The Notion of Norm in Spoken French. Production and Perception". In Detey, S., Durand, J., Laks, B. \& Lyche, C. (dir.). Varieties of Spoken French. Cambridge: Oxford University Press, pp. 55-67.

Detey, S., Racine, I., Kawaguchi, Y. \& Eychenne, J. (2016c). La prononciation du français dans le monde : du natif à l'apprenant. Paris : CLE International.

Detey, S., Durand, J., Laks, B. \& Lyche, C. (2010). Les variétés du français parlé dans l'espace francophone. Ressources pour l'enseignement. Paris : Ophrys.

Didelot, M. \& Racine, I. (sous presse). « Pour "être intelligible" en français langue étrangère, doiton s'exprimer 'sans accent'?». Cahiers de linguistique.

Doerr, N. M. (2009). "Investigating "Native Speaker Effects": Toward a New Model of Analysing "Native Speaker" Ideologies". In Doerr, N. M. (dir.). The Native Speaker Concept. Berlin: Mouton de Gruyter, pp. 15-46.

Eckert, P. (2008). "Variation and the Indexical Field". Journal of Sociolinguistics, vol. 12, pp. 453-476.

Field, J. (2005). "Intelligibility and the Listener: The Role of Lexical Stress". TESOL Quarterly, vol. $39, n^{\circ} 3$, pp. 399-423.

Fischer, C. (2007). « L'évaluation des compétences orales d'étudiants en formation à l'enseignement ». In Plessis-Bélair, G., Lafontaine, L. \& Bergeron, R. (dir.). La didactique du français oral au Québec : recherches actuelles et applications dans les classes. Québec : Presses de l'Université du Québec, pp. 220-243.

Gass, S. \& Varonis, E. (1984). "The Effect of Familiarity on the Comprehensibility of Nonnative Speech". Language Learning, vol. 34, pp. 65-87.

Grin, F. (1999). Compétences et récompenses. La valeur des langues en Suisse. Fribourg : Editions universitaires.

Guess, R., Lyche, C. \& Meisenburg, T. (2012). Phonological Variation in French. Illustrations from Three Continents. Amsterdam/Philadelphia : John Benjamins.

Hambye, P. \& Romainville, A.-S. (2014). Apprentissage du français et intégration. Des évidences à interroger. Bruxelles : E.M.E.

Harding, L. (2017). "What Do Raters Need in a Pronunciation Scale? The User's View". In Isaacs, T. \& Trofimovich, P. (dir.). Second Language Pronunciation Assessment: Interdisciplinary Perspectives. Bristol: Multilingual Matters, pp. 12-34.

Hogan-Brun, G., Mar-Molinero, C. \& Stevenson, P. (2009). Discourses on Language and Integration: Critical Perspectives on Language Testing Regimes in Europe. Amsterdam/Philadelphia: John Benjamins.

Horner, D. (2014). "And What about Testing Pronunciation? A Critical Look at the CEFR Pronunciation Grid and a Proposal for Improvement". In Rupp, L. \& van den Doel, R. (dir.). Pronunciation Matters. Accents of English in the Netherlands and Elsewhere. Amsterdam : University Press, pp. 109-124.

Houdebine-Gravaud, A.-M. (2017). « Brève histoire d'une norme prescriptive : le centralisme linguistique ». In Feuillard, C. (dir.). Usage, Norme et Codification : de la diversité des situations à l' utilisation du numérique. Louvain-la-Neuve : E.M.E., pp. 19-32.

Irvine J. (2001). "'Style' as Distinctiveness: The Culture and Ideology of Linguistic Differentiation". In Eckert, P. \& Rickford, J. (dir.). Style and Sociolinguistic Variation. New-York: Cambridge University Press, pp. 21-43. 
Isaacs, T. (2016)."Assessing Ppeaking". In Tsagari, D. \& Banerjee, J. (dir.). Handbook of Second Language Assessment. Berlin: DeGruyter Mouton, pp. 131-146.

Jenkins, J. (2000). The Phonology of English as an International Language. Oxford: Oxford University Press.

Jodelet, D. (1989). Représentations sociales. Un domaine en expansion. Paris : PUF.

Kang, O. \& Rubin, D. (2009). "Reverse Linguistic Stereotyping: Measuring the Effect of Listener Expectations on Speech Evaluation". Journal of Language and Social Psychology, vol. 28, $\mathrm{n}^{\circ}$ 4, pp. 441-456.

Kennedy, S., Blanchet, J. \& Guénette, D. (2017). "Teacher-Raters'Assessment of French Lingua Franca Pronunciation". In Isaacs, T. \& Trofimovich, P. (dir.). Second Language Pronunciation Assessment: Interdisciplinary Perspectives. Bristol: Multilingual Matters, pp. 210-236.

Lauret, B. (2007). Enseigner la prononciation du français: questions et outils. Paris : Hachette.

Lavoie, C. \& Bouchard, E. (2017). « Formation universitaire à l'évaluation de l'oral : regard sur la capacité d'autoévaluation de futurs enseignants ». In De Pietro, J.-F., Fischer, C. \& Gagnon, R. (dir.). L'oral aujourd'hui : perspectives didactiques. Namur : Presses Universitaires de Namur, pp. 259-274.

LeVelle, K. \& Levis, J. (2014). "Understanding the Impact of Social Factors on L2 Pronunciation: Insights form Learners". In Levis, J.-M. \& Moyer, A. (dir.). Social Dynamics in Second Language Accent . Berlin: DeGruyter Mouton, pp. 97-118.

Lipiansky, M. (1991). « Représentations sociales et idéologies: analyse conceptuelle ». In Aebischer, V., Deconchy, J.-P. \& Lipiansky, M. (dir.). Idéologies et représentations sociales. Cousset : DelVal, pp. 35-63.

Lippi-Green, R. (2012) [1997]. English with an Accent. Language, Ideology, and Discrimination in the United States. New York/London: Routledge ( $2^{\text {nd }}$ ed.).

Lyche, C. (2010). «Le français de référence : éléments de synthèse ». In Detey, S., Durand, J., Laks, B. \& Lyche, C. (dir.). Les variétés du français parlé dans l'espace francophone. Ressources pour l'enseignement. Paris : Ophrys, pp. 143-165.

Munro, M. J. \& Derwing, T. M. (2006). "The Functional Load principle in ESL Pronunciation Instruction: An Exploratory Study". System, vol. 34, $\mathrm{n}^{\circ}$ 4, pp. 5P0-531.

Piccardo, E. (2016). Common European Framework of Reference for Languages: Learning, Teaching, Assessment. Phonological Scale Revision Process Report. Disponible en ligne. https://rm.coe.int/ phonological-scale-revision-process-report-cefr/168073fff9 (Consulté le 29 juin 2018).

Pickering, L. (2006). "Current Research on Intelligibility in English as a Lingua Franca". Annual Review of Applied Linguistics, vol. 26, pp. 219-233.

Pöll, B. (2005). Le français, langue pluricentrique? Etudes sur la variation diatopique d'une langue standard. Frankfurt am Main : Peter Lang.

Prikhodkine, A. \& Correia Saavedra, D. (2016). « Pratiques langagières légitimes et ethnicité : quels rapports? ». In Arrighi, L. \& Boudreau, A. (dir.). Langue et légitimation: La construction du locuteur francophone. Québec : Les Presses de l'Université Laval, pp. 107-126.

Rajagopalan, K. (2010). "The Soft Ideological Underbelly of the Notion of Intelligibility in Discussions about 'World Englishes'". Applied Linguistics, vol. 31, n 3, pp. 465-470.

Rubin, D. (1992). "Nonlanguage Factors Affecting Undergraduates' Judgments of Nonnative English-Speaking Teaching Assistants". Research in Higher Education, vol. 33, nº 4, pp. 511-531. 
Smith, L. E. \& Nelson, C. (1985). "International Intelligibility of English: Directions and Resources". World Englishes, vol. 4, pp. 333-342.

Trottier, S. (2007). L'évaluation de la production orale chez les adultes en francisation: analyse des critères de cinq enseignantes. Mémoire de maîtrise en linguistique, Université du Québec, Montréal.

Van Berten, C. (2016). «L'enseignement du français dans les universités américaines: entre mondialisation et contextualisation ». Cahiers AFLS, vol. 20, n 2, pp. 19-39.

\section{NOTES}

1. Par commodité, le terme «L2 » est utilisé pour désigner toute langue apprise en plus de la langue première, qu'il s'agisse de la première, deuxième ou énième langue.

2. À titre d'exemple, on peut mentionner la récente opération Papyrus, menée dans le canton de Genève, visant à régulariser la situation d'étrangers en situation irrégulière et dans le cadre de laquelle les exigences en termes de langue (niveau A2 de français requis) sont uniquement évaluées sur la base d'un entretien oral (https://www.ge.ch/document/bie-operation-papyrusconditions-procedure-depot-demande-normalisation/telecharger).

3. «le plus grand obstacle à une communication réussie » (notre traduction).

4. Nous entendons par accent - et, par extension, parole accentuée - un faisceau de traits segmentaux et prosodiques distribués socialement, géographiquement, etc. (Lippi-Green, 2012 : 45).

5. «[...] la figure du locuteur natif réapparaît subrepticement, cette fois-ci par la petite porte, et cela de manière bien trop furtive » (notre traduction).

6. Autrement dit, une prononciation qui correspondrait à l'idéal - fantasmé probablement - d'un standard non marqué.

7. Le récent ouvrage de Detey et al. (2016c) - qui décrit, sur une base expérimentale, les principales difficultés de prononciation d'apprenants de 19 L1 différentes - constitue un premier pas vers ce type d'inventaire précis pour le français.

8. Les éléments synthétisés par Horner (2014 113) sont les suivants : "word stress and associated phonemic realizations, with consonants being more important than vowels/diphthongs, and sentence stress. [...]. Non-core elements would then include: weak forms, contractions, rhythm, prosody and to a lesser extent, intonation " («accent de mot et réalisations phonémiques associées, dans lesquelles les consonnes sont plus importantes que les voyelles ou les diphtongues, et que l'accent de phrase. [...] Les éléments secondaires seraient par conséquent les formes faibles, les contractions, le rythme, la prosodie et, dans une moindre mesure, l'intonation ", notre traduction).

9. À ce titre, on peut mentionner plus spécifiquement l'inventaire des voyelles avec une norme locale différente pour cinq grandes régions (Belgique, Suisse romande, Midi de la France, Afrique centrale et Canada) proposé dans Detey et al. (2016b : 60).

\section{RÉSUMÉS}

Avec la globalisation poussée du monde actuel, de nouveaux enjeux ont émergé autour de l' évaluation de la compétence orale, dans laquelle la composante phonique tient une place 
prépondérante. Dans cette contribution, nous proposons une réflexion autour des notions d' intelligibilité - objectif annoncé de l'enseignement/apprentissage de la prononciation aujourd' hui - et de parole accentuée, au regard de leur utilisation dans le descripteur de la compétence phonologique du CECRL, récemment remanié. Si cette révision constitue indéniablement une amélioration, la capacité à dissocier « force de l'accent » et « intelligibilité » nécessite néanmoins un effort important à fournir en termes de formation des enseignants et évaluateurs.

In an increasingly globalised world, new challenges have emerged relating to the evaluation of oral language competence. The phonetic component holds a dominant place here, and this paper constitutes a reflection around two notions, i.e. intelligibility - an upcoming objective of modern pronunciation teaching -, and accented speech, in light of their place and usage in the descriptor of phonological competence in CEFRL (Common European Framework of Reference for Languages). We compare the old and the recently revised version of the descriptor, and although the latter undeniably constitutes an improvement, much work still needs to be done when it comes to pronunciation and the training of teachers and assessors.

\section{INDEX}

Mots-clés : enseignement de la prononciation, intelligibilité, accent, CECRL, évaluation.

Keywords : L2 pronunciation teaching, intelligibility, foreign accent, CEFRL, L2 pronunciation assessment.

\section{AUTEURS}

\section{MARION DIDELOT}

École de langue et de civilisation françaises, Université de Genève Marion Didelot est assistante-doctorante à l'École de langue et de civilisation françaises (ELCF) à l'Université de Genève. Ses recherches portent sur l'enseignement de la prononciation du Français Langue Étrangère (FLE) et sur la perception et la réception de la parole accentuée native et non native.

Marion.Didelot@unige.ch

\section{ISABELLE RACINE}

École de langue et de civilisation françaises, Université de Genève Isabelle Racine est professeure à l'Ecole de Langue et de Civilisation Françaises (ELCF) à l'Université de Genève. Ses recherches portent sur l'acquisition phonologique d'une langue étrangère, en particulier sur les difficultés posées par la variation phonique dans l'apprentissage du FLE et la place de la composante phonique au sein de la production orale, en lien avec les enjeux de cette compétence orale. Elle codirige les projets « Interphonologie du français contemporain (IPFC, http://cblle.tufs.ac.jp/ipfc/) et « Phonologie du Français Contemporain (PFC, http://www.projet-pfc.net) et est également coéditrice du volume La prononciation du français dans le monde : du natif à l'apprenant, paru en 2016.

Isabelle.Racine@unige.ch

\section{FRANÇOISE ZAY}

École de langue et de civilisation françaises, Université de Genève

Françoise Zay est chargée d'enseignement à l'École de Langue et de Civilisation Françaises (ELCF) 
à l'Université de Genève. Après s'être intéressée aux corpus oraux et aux aspects syntaxiques du français parlé, elle collabore à certains des projets de recherche menés par Isabelle Racine sur l'acquisition phonologique d'une langue étrangère, notamment dans le cadre d'IPFC.

Françoise.Zay@unige.ch

\section{ALEXEI PRIKHODKINE}

École de langue et de civilisation françaises, Université de Genève

Alexei Prikhodkine est chargé d'enseignement à l'Ecole de Langue et de Civilisation Françaises (ELCF) à l'Université de Genève. Ses recherches portent sur la perception de la variation sociolinguistique par des locuteurs natifs et non natifs ainsi que sur le lien entre langue(s) et intégration, notamment pour des populations migrantes. Il est co-éditeur du volume Response to Language Varieties. Variability, processes and outcomes, paru en 2015.

Alexei.Prikhodkine@unige.ch 